\title{
Alzheimer's Disease Classification Using Deep CNN
}

\section{Shikha Agrawal, Neha Sunil Pandharkar, Pooja Arvind Khandelwal, Pratiksha Ashok Pandhare, Janhavi Sanjay Deoghare}

Computer Department, AISSMS Institute of Information Technology, Pune, Maharashtra, India

\section{ABSTRACT}

Article Info

Volume 7, Issue 3

Page Number: 325-331

\section{Publication Issue :}

May-June-2021

\section{Article History}

Accepted : 15 May 2021

Published : 22 May 2021
Especially in the world, the deep learning algorithm has become a technique of choice for analyzing medical images rapidly. Alzheimer's disease (AD) is regarded to be the most prevalent cause of dementia, and only 1 in 4 individuals with Alzheimer's are estimated to be diagnosed correctly on time. However, there is no refractory available treatment, the disorders can be managed when the loss is still mild and the treatment is most effective when it is initiated before significant downstream damage, i.e. mild cognitive impairment (MCI) or earlier steps. Physiological, neurological analysis, neurological and cognitive tests are clinically diagnosed with AD. A better diagnostic needs to be developed, which is addressed in this paper. We concentrate on Alzheimer's disease in this article and discuss different methods are available to detect Alzheimer's. Reviewed the different data sets available for studying data on Alzheimer's disease and finally comparing appropriate work done in this area.

Index Terms : Machine Learning, Deep Learning, Alzheimer's Disease, Convolution Neural Network. MR Images.

\section{INTRODUCTION}

Alzheimer's disease (AD) is a neurological disease that impacts people and is regarded to be the most prevalent cause of dementia, though other illnesses and circumstances may also cause dementia. Patient memory is described by decline, ability to prepare and use language, problem solving, and other cognitive abilities, and these features influence a person's capacity to do daily tasks. This decline happens in human capacity because in areas of the brain involved in cognitive function, nerve cells (neurons) have been damaged and do not usually perform any function.Neuronal damage in
Alzheimer's disease eventually influences those components of the brain that allow an individual to perform fundamental physical tasks such as walking and swallowing. Alzheimer's disease is an incurable disease that has not yet been treated[1]. Dementia is a paragliding word used to define a number of diseases, and there are many distinct kinds of dementia, including Alzheimer's disease, vascular dementia, lewy-body dementia, and others, but Alzheimer's Type Dementia (AD) is far away, the most prevalent cause of dementia, and there is a relationship to this type of dementia. It is estimated that 5.3 million U.S. people have Alzheimer's disease, according to the 2015 Alzheimer's Report[2]. It is costly to monitor 
the progress of the disease over time, even if the disease is correctly diagnosed. Treatment is regarded to be the most efficient when it is initiated before important drift loss, i.e. before Alzheimer's disease diagnosis, or even earlier in the first stage of light cognitive deficiency (LCI).

Early detection of dementia is commonly recognized as being restricted to more efficient intervention and morbidity (Peterson et al.[4]). They also conclude from their job that those who fulfill the MCI requirements can be separated from those with good areas of control and very benign Alzheimer's disease. This group of subjects is a diagnostic unit that can be attributed to the involvement in the treatment. To date, most types of mental illnesses have been diagnosed on the basis of clinical observation. In particular, it includes identifying the symptoms grouped together, the time of symptoms, and their tendency to be repetitive or tend to be outdated. There is no cure for Alzheimer's disease at present as well as no type of reliable and efficient original diagnostic techniques available. Boise et al.[3] Clinical assessment and confirmation of past low diagnostic rates research and explain a possible explanation for it, combined with the subtlety of symptoms of psychiatrists in their clinical practice they do. Physical and neurological examinations are used to diagnose Alzheimer's disease, and other intellectual deficiency symptoms are checked by standard neurological and cognitive tests. The overall strategy is based on elimination diagnosis, i.e. until Alzheimer's disease is the last choice, all else does the decision. According to Dubois et al.[6] guidelines for the diagnosis of Alzheimer's disease, in relation to the above-mentioned clinical remedies, emphasize the role played by using different biomarkers. These include the measurement of protein profiles of magnetic resonance imaging (MRI), positron emission tomography (PET), cerebrospinal fluid $(\mathrm{CSF})$, and genetic risk profile analysis, although these are costly and hard to scale on a big amount of evaluations. Obviously, diagnosing Alzheimer's disease needs a good diagnostic tool to be developed, potentially using the methods of data mining and data analysis. If new drugs or prevention techniques were created and demonstrated efficient, then an original diagnosis could allow intervention in the first phase, which would have demonstrated advantages, yet we are at a moment when diagnosis is made using only the signs and symptoms of the disease, and this is difficult. There is no single test that can demonstrate whether or not an individual has Alzheimer's disease. Although therapists can almost always determine if an individual has dementia, determining the precise cause can be extremely difficult. Alzheimer's diagnosis needs thorough clinical examination, including:

- Input from any individual or relative of an individual in the family about improvements in their cognitive ability or behaviour; mental status testing;

- Physical and neurological examination;

- Testing (such as blood testing and/or brain imaging) may detect dementia symptoms such as other causes of cancer that may explain the person's symptoms.

- A physician can diagnose Alzheimer's disease and requires abilities, knowledge and experience by gathering the performance from the above evaluation set. None of which can be replicated readily.

\section{METHODS}

There are two kinds of computer-aided detection using brain therapy images to diagnose $A D$ in the early phases, depending on the pattern recognition issue: Pattern Classification and Facility Extraction. The image of the brain reflects distinct phases of dementia or pattern during the application training stage. To recognize these patterns, quantitative image 
analysis is used. Before the training stage, all feeding dimensional features are recognized or controlled, it is recognized and fed in some particular algorithms[7]. Typically trained phase is regarded "black box technique." Second, characteristics are removed, paired or chosen to predict class labels[8] and similarly classifiers are used to produce a classification that demonstrates the disease stage. In order to recognize Alzheimer's disease, we will concentrate on state-of-the-art facilities extraction methods and classification methods.

The feature extraction technique was categorized into three ways to obtain characteristics from brain image datasets: voxel-based, ROI-based, and patchedbased[9].

\section{Voxel-Based Approaches}

Voxel-based method is a method of neuroimaging. It enables statistical strategies to statistical mapping to study focal differences diagnosis. In morphometry, by determining brain scanning and counting regions of interest on brain images, the volume of brain or subtype is calculated. For each model, Voxel-based morphometry (VBM) will register, giving individuals a definite concept of brain disease. Ultimately, the volume of the brain in each voxel is compared. For each model, Voxel-based morphometry (VBM) will register, giving individuals a definite concept of brain disease. Ultimately, the quantity of brain in each vowel is compared. VBM is unable to distinguish the small volume difference. Identification of MRI and PET images score correlation and diagnostic status. ADAS-Cog's level of precision was 716\% and MMSE $655 \%$.

\section{Region of Interest (Roi) -Based Approaches}

The region of interest-based strategy is used for image characteristics and it will classify several portions of the brain component, such as the corpus callosum, better longitudinal fascia, hippocampi, fasicus, and singulam. Pathological trials of
Alzheimer's disease neurodegeneration will occur in the human lobe and limbic system, hippocampus and neocortical areas[15].

In many areas of $\mathrm{AD}$, hippocampsi atrophy is used as a marker. Selected characteristics from brain images include the size or quantity or weighted combination of hippocampsi. ROI segmentation happens prior to the application of feature extraction method in the ROI-based approach[16].

\section{Patch-Based Approach}

For image representation and recovery, a patch-based method is suggested. This technique is used mainly in the varying selection of each picture component for the point-wise selection of the specified image patch of the precise size. Within the adaptively specified information, there is some sample weight in each pixel. In medical research, this strategy will demonstrate optimistic outcomes.

The hippocampal atrophy rate from the MRI image is discussed by Josephine Barnes et al.[28 ]. This rate of atrophy is helpful to track and diagnose Alzheimer's disease patients. Furthermore, it addressed the metaregressions and meta-analysis of the specified datasets. Average rates were discovered at 4.66\% (95\%).

\section{DEEP LEARNING NETWORKS}

Several common deep learning networks like CNN models are addressed in this paper. Since deep learning has been increasing very rapidly, however, many new networks and architectures appear over the next few months, which is out of this paper's scope.

\section{A. Convolutional Neural Network (CNN)}

Also, CNN is a commonly used deep learning algorithm [5]. CNN has three primary benefits: sharing parameters, sparse interactions, and representations of equivalents. Local connections and 
shared weights are used in the network rather than traditional fully linked networks to fully utilize the two-dimensional structure of an input information (e.g. image signals). This method consists of very few parameters, making the network simpler to train and quicker. This procedure is comparable to that in the cells of the visual cortex. These cells are more susceptible than the whole scene to small parts of a scene. There are a number of convolutional layers in typical CNNs followed by pooling (subsampling) layers, and generally fully-connected layers (like Multilayer Perceptron (MLP)) are used in the final phase layers. Figure 1 demonstrates an illustration of an image classification CNN architecture. The layers in CNNs have three-dimensional inputsx arranged, $\mathrm{m}$ ${ }^{*} \mathrm{~m}{ }^{*} \mathrm{r}$, where $\mathrm{m}$ refers to the input height and width, and $r$ refers to the depth or channel numbers (e.g., $r=3$ for an RGB image). There are many filters (kernels) $\mathrm{k}$ of size $\mathrm{n}$ as well as $\mathrm{n}$ as $\mathrm{q}$ for each convolutional layer. In this case, $\mathrm{n}$ should be smaller than the image input, but q can either be smaller or the same size as r.As previously stated, filters are the basis of local links that are converted to input and share the same parameters (weight $\mathrm{W} \mathrm{k}$ and bias $\mathrm{b}^{\wedge} \mathrm{k}$ ) to produce $k$ feature maps $\left(h^{\wedge} k\right)$, each one of size $m-$ $\mathrm{n}$ - 1. Similar to MLP, the convolutional layer calculates a dot product between the weights and their outputs (as shown in Equation (1)), but the outputs are tiny areas of the initial quantity of input. Then an activation feature (f) or a nonlinearity is applied to the convolution layers output.

\section{$\mathbf{h}^{\wedge} \mathbf{k}=\mathbf{f}\left(\mathrm{W}^{\wedge} \mathbf{k} * \mathbf{x}+\mathrm{b}^{\wedge} \mathbf{k}\right)$}

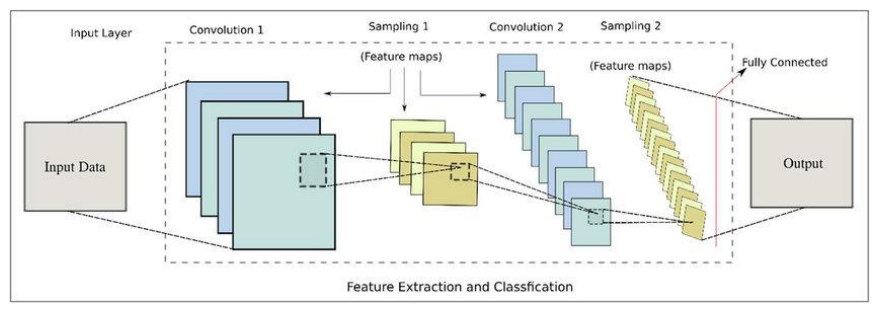

Figure 1 : Demonstrates an illustration of an image classification $\mathrm{CNN}$ architecture.
The two popular architectures that used in image classification techniques.

\section{VGG16:}

VGG16 is an Oxfords Visual Geometry Group (VGG) 16-layer network[6]. In ILSVRC-2014, it participated in the ImageNet competition. One of the primary reasons VGG16 won the contest is that, by pushing to 16-19 layers and using very small $(3 \times 3)$ convolution filters, it is one of the first network architecture to explore network depth.

\section{Inception}

The architecture of Inception is a version of Google's constructed profound learning framework. There have been distinct iterations over the years. Inception V4 was implemented in [7], mentioned in this paper. The breakthrough of the Inceptions is in the realization that it is possible to learn non-linear features by altering how convolutional layers are linked. The fully connected layer is therefore removed in preference to a worldwide average pooling that averages the function maps and then connects for classification with a softmax layer. There are therefore fewer parameters, resulting in less overfitting.

\section{CLASSIFICATION TECHNIQUE}

This section discusses briefly multiple methods of classification used to categorize the diseases. It was possible to train all the classifier method and then apply it to discover a test case diagnosis. The widely used method for classifying Alzheimer's disease.

\section{A. Random Forest (RF)}

As an ensemble learning technique for regression, classification, and other activities, random forests or random decision forests are used. It builds a variety of 
decision trees at the moment of practice and provides the output of the class which is the mode of the individual trees ' mean regression) or classes (classification). Compared to the other classification methods, it provides better results.

Tong Tong et al.[8] discussed a number of significant variables such as age correction, precision of registration, selection of features and training data selection. It also addressed novel grading biomarkers for the transformation of MCI-AD and used a sparse method of representation with Random Forest. Finally, the accuracy of the classification enhanced from $62 \%$ to $72 \%$.

\section{B. Support Vector Machine (SVM)}

SVM plays an important role in the development of machine learning model. It is a controlled model of learning machines and their related study algorithms of learning. Processed information is used to analyze and classify regression or other tasks. SVM, with finite samples of practice, is mostly used to fix the issue of pattern classification. The method of classification has been categorized into two primary steps.

Xiaohong W. Gao et al.[5] implemented an unattended automatic computer that assisted the CNN process on Alzheimer's disease segmentation, classification and measurement. The 95.6 percent accuracy value was accomplished. $\mathrm{AD}$, lesion, and normalcy classification accomplished an accuracy of $88 \%, 76.6 \%$, and $95 \%$ respectively.

Deep learning architecture (LeNet) was discussed by Saman Sarraf et al.[13] to train and test huge picture information. CNN and LeNet-5 architectures are used to classify fMRI, MRI, and state functional magnetic resonance imaging (rs-fMRI) information for clinical scanning to acknowledge AD. The 96.85 percent accuracy value was obtained.

\section{K-Nearest Neighbor}

K-nearest neighbors ( $\mathrm{k}-\mathrm{NN}$ ) are a non-parametric technique and are algorithms for data mining. KNN used for image recognition domain regression and classification. The input comprises of the $\mathrm{k}$ nearest training data in the feature space in both regression and classification instances. The output will be whether $\mathrm{k}-\mathrm{NN}$ based on the information is used for classification or regression.

By using the EEG function, Charlotte Cecere et al.[15] addressed the approach to machine learning and then contrasted various classification methods. These techniques are algorithms such as SVM, KNN, CART trees, naïve Bayes and AdaBoost. The highest accuracy of SVM was 78.5 percent.

Abdalla R. Gad et al.[18] suggested automatic classification of subjects from MRI pictures and two methods of classification. The first is $\mathrm{KNN}$ and the second is SVM. The author identified the class of the chosen function for which one precision is greater. KNN and SVM achieved the highest average precision rate of 95,84 percent and 97,92 percent.

\section{Decision Tree (DT)}

Decision trees are just a straightforward yet strong technique of classification. Decision trees are generated by algorithms identifying different methods to divide a dissemination of information into branch-like fragments. These fragments constitute an inverted tree of choice of which the root node is at the top of the tree. CART (Classification and Regression Tree), ID3 (Iterative Dichotomized 3) and C4.5 are examples of decision tree algorithms. These algorithms vary among several other variations in how characteristics are chosen in the tree as dividing nodes.

Dana el at. [19] proposed the decision tree provided corresponding to the sample information. Selection of nodes in the tree computed for each attribute on the 
Entropy or Information Gain. The correct attribute is selected as a dividing attribute for each tree level if it provides us the greatest gain of information. Depending on the attribute values of the fresh patients, the decision tree is traveled downwards from either the root node until a leaf node is reached. The leaf node reflects the forecast of the state of Alzheimer's disease.

\section{DATASETS}

\section{A. Alzheimer's disease Neuroimaging Initiative (ADNI)}

The Alzheimer's disease Neuroimaging Initiative (ADNI) is a collaboration of universities and medical centers formed in the United States and Canada to create specific imaging methods and clinical trial processes in ordinary topics, subjects with MCI, and subjects with moderate Alzheimer's disease.

The main objectives of ADNI are to create enhanced techniques leading to standardized norms for the acquisition of longitudinal, multisite MRI and PET information on patients with $\mathrm{AD}$, patients with MCI and elderly controls, to create an affordable data repository describing longitudinal changes in brain structure and metabolism while obtaining parallel clinical information.

Diagnostic group (mean and SD for quantitative measures, percentage for categorical factors) analyzed the basic features of the respondents. At average, group features were contrasted with non-parametric experiments (Kruskal-Wallis) for quantitative measurements, using various Hochberg comparison procedure to compare means, and using $\mathrm{X}^{\wedge} 2$ or accurate tests to compare ratios.

Change over time has been explained in three different ways: conversion ratio, mean or percentage difference in quantitative measurement among the baseline and 12-month follow-up, and annual change rate estimated from combined effect regression models for quantitative measurement using all accessible follow-up data. The Alzheimer's disease Neuroimaging Initiative (ADNI) unites researchers with survey data as they operate to define Alzheimer's disease development. The objective of ADNI scientists is to gather, validate and use information such as images of MRI and PET, genetics, cognitive testing, CSF and blood biomarkers as disease predictors [21-23].

\section{GENERAL PROCEDURE OF IMAGE CLASSIFICATION}

There are seven steps we are going to consider for building images classification. All seven steps mention as follows [27].

A. Data Collection : The quantity \& quality of your data dictate how accurate our model is. The outcome of this step is generally a representation of data which we will use for training Using pre-collected data, by way of datasets still fits into this step.

B. Data Preparation : Wrangle data and prepare it for training Clean that which may require it (remove duplicates, correct errors, deal with missing values, normalization, data type conversions, etc.) Randomized data, which erases the effects of the particular order in which we collected and/or otherwise prepared our data Visualize data to help detect relevant relationships between variables or class imbalances (bias alert!), or perform other exploratory analysis Split into training and evaluation sets

C. Choose a model : Different algorithms are for different tasks; choose the right one as per requirement.

D. Train the model : The goal of training is to answer a question or make a prediction correctly as often as possible Linear regression example: algorithm would need to learn values for $\mathrm{m}$ (or $\mathrm{W}$ ) and $\mathrm{b}$ ( $\mathrm{x}$ is input, $\mathrm{y}$ is output) each iteration of process is a training step. 
E. Evaluate the model : Uses some metric or combination of metrics to "measure" objective performance of model. Test the model against previously unseen data. This unseen data is meant to be somewhat representative of model performance in the real world, but still helps tune the model (as opposed to test data, which does not) Good train/eval split? $80 / 20,70 / 30$, or similar, depending on the domain, data availability, dataset particulars, etc.

F. Parameter tuning : This step refers to hyper parameter tuning, which is an "artform" as opposed to a science Tune model parameters for improved performance Simple model hyper parameters may include: number of training steps, learning rate, initialization values and distribution, etc.

G. Make Predictions : Using further (test set) data which have, until this point, been withheld from the model (and for which class labels are known), are used to test the model; a better approximation of how the model will perform in the real world.

\section{CONCLUSIONS}

A detailed survey and overview of computer-aided dementia diagnosis from the classification of patterns can be divided into two main parts: the method of extraction of features and the method of classification. Extraction feature, including SVM, Random Forest, Decision Tree and KNN, based on Voxel, ROI, and Patch. Review the different datasets are also accessible. The comparison section shows the diagnosis of Alzheimer's disease (AD), Mild Cognitive Impairment (MCI) and Elderly Normal Control (NC) with newly developed algorithms accomplished high accuracy.

\section{REFERENCES}

[1]. Z. Alom et al., "A State-of-the-Art Survey on Deep Learning Theory and Architectures," pp. 167, 2019.
[2]. N. M. Khan and M. Hon, "Transfer Learning with intelligent training data selection for prediction of Alzheimer's Disease," Neurocomputing, vol. 7, pp. 72726-72735, 2018.

[3]. C. Feng et al., "Deep Learning Framework for Alzheimer's Disease Diagnosis via 3D-CNN and FSBi-LSTM," IEEE Access, vol. 7, pp. 1-1, 2019.

[4]. E. Jabason, M. O. Ahmad, and M. N. S. Swamy, "Shearlet based Stacked Convolutional Network for Multiclass Diagnosis of Alzheimer's Disease using the Florbetapir PET Amyloid Imaging Data," 2018 16th IEEE Int. New Circuits Syst. Conf. NEWCAS 2018, pp. 344-347, 2018.

[5]. F. Li, D. Cheng, and M. Liu, “Alzheimer's disease classification based on combination of multimodel convolutional networks," IST 2017 - IEEE Int. Conf. Imaging Syst. Tech. Proc., vol. 2018Janua, no. 61375112, pp. 1-5, 2018.

[6]. D. Cheng and M. Liu, "Combining convolutional and recurrent neural networks for Alzheimer's disease diagnosis using PET images," IST 2017 IEEE Int. Conf. Imaging Syst. Tech. Proc., vol. 2018-Janua, no. Mci, pp. 1-5, 2018.

[7]. A. Farooq, S. Anwar, M. Awais, and S. Rehman, "A deep CNN based multi-class classification of Alzheimer's disease using MRI," IST 2017 - IEEE Int. Conf. Imaging Syst. Tech. Proc., vol. 2018Janua, pp. 1-6, 2018.

\section{Cite this article as :}

Shikha Agrawal, Neha Sunil Pandharkar, Pooja Arvind Khandelwal, Pratiksha Ashok Pandhare, Janhavi Sanjay Deoghare, "Alzheimer's Disease Classification Using Deep CNN ", International Journal of Scientific Research in Computer Science, Engineering and Information Technology (IJSRCSEIT), ISSN : 2456-3307, Volume 7 Issue 3, pp. 325-331, May-June 2021. Available at

doi : https://doi.org/10.32628/CSEIT217371

Journal URL : https://ijsrcseit.com/CSEIT217371 